\title{
Overdominant lethals as part of the conifer embryo lethal system
}

\author{
CG Williams, LD Auckland, MM Reynolds and KA Leach ${ }^{1}$ \\ Graduate Genetics Program, Texas AEM University, 305 Horticulture Building, TAMU 2135, College Station, TX 77843-2135, USA
}

In pines, self-pollination rates can be as high as 34\% yet only $5 \%$ of viable seed is a product of self-fertilization. This decline in selfed seed viability is the consequence of post-fertilization exclusion mechanisms operating via the embryo lethal system. Recent molecular marker dissection studies suggest that the embryo lethal system is composed of semilethal factors dispersed across the genome, but it is not clear whether overdominant lethal factors are rare or representative. The study objective was to determine if overdominance was rare for the embryo lethal system in conifers. Three cohorts of selfed offspring from a single Pinus taeda parent were genotyped for nuclear microsatellites. Maximum likelihood tests based on distorted segregation ratios for single markers and for interval mapping were used to infer the degree of dominance. Four hypotheses about overdominance lethal factors were tested: (1) overdominant lethal factors rarely occur within the embryo lethal system, (2) overdominant lethal factors are rarely detected because they are transient and display stage-specific expression, (3) overdominant lethal factors are rarely detected due to tight linkage with rare marker alleles and (4) dominance estimation is unbiased by gametic selection. Four out of the seven chromosomal segments were linked to an overdominant lethal factor. One of these four segments had symmetric overdominance, an effect which persisted from embryo maturity through germination. Four overdominant lethal factors were linked to common and rare marker alleles. Gametic selection was not a source of bias in dominance estimation. Overdominant or pseudooverdominant lethal factors are a common component of the conifer embryo lethal system.

Heredity (2003) 91, 584-592, advance online publication, 17 September 2003; doi:10.1038/sj.hdy.6800354

Keywords: prezygotic selection; pseudo-overdominance; balanced lethals; selfing; Pinus taeda L.; gymnosperms

\section{Introduction}

Self-pollination rates can be as high as $34 \%$ in the lower crown; yet only $5 \%$ of viable seed is a product of selffertilization in Pinus taeda (Franklin, 1969). The embryo lethal system selects against self-pollinated embryos soon after fertilization (Bramlett and Popham, 1971; Koski, 1971) although little is known about the genetic mechanism(s) underlying the system. The system has long been considered to have polygenic inheritance because biometrical models yield estimates of loci numbers ranging from 75 to 10000 (Koski, 1971; Namkoong and Bishir, 1987; Williams and Savolainen, 1996).

The advent of molecular markers has provided a valued alternative to biometrical models; with molecular markers, the embryo lethal system can be dissected into chromosomal segments so that mode(s) of inheritance can be characterized.

Recent marker studies suggest that the embryo lethal system is composed of independent semilethal factors dispersed throughout the genome (Kuang et al, 1999; Remington and O'Malley, 2000a). Support has been limited for epistatic or synergistic models (Griffin and

Correspondence: CG Williams, Texas AEM University, 305 Horticulture Building, TAMU 2135, College Station, TX 77843, USA.

E-mail: claire-williams@tamu.edu

${ }^{1}$ Current address: Genetics Program University of Missouri, Columbia, MO, USA

Received: 21 May 2002; accepted: 27 June 2003
Lindgren, 1985; Remington and O'Malley, 2000a,b). Lethal factors expressed early in embryo development do not contribute to inbreeding depression for older growth traits (Remington and O'Malley, 2000b).

The crux of the question is whether overdominant effects are rare for the embryo lethal system. Overdominance predicts heterozygote superiority relative to both homozygotes. Partial dominance, thought to be more prevalent in the embryo lethal system, predicts inbreeding depression from an increased expression of deleterious recessive alleles as a result of increased homozygosity. The degree of dominance $h$ must be estimated experimentally. It measures the extent to which the deleterious allele is expressed in the heterozygote.

Apparent overdominance can be attributed to deleterious recessive alleles linked in repulsion. This is defined as pseudo-overdominance (Mangelsdorf, 1952; Stuber et al, 1992). Pseudo-overdominance is likely to occur if lethal factors are clustered or dense in certain chromosomal segments. For molecular markers $A$ and $B$, a balanced lethal complex $A-L_{1}-b-l_{2} / a-l_{1}-B-L_{2}$ would have two cryptic lethal factors $l_{1}$ and $l_{2}$ linked in repulsion. In this case, marker heterozygotes will be over-represented and marker homozygotes will be under-represented or absent. Using fine-scale mapping, each deleterious allele would exert a partially dominant effect.

Estimating fitness values for each genotypic class also requires estimates of the selection coefficient $s$, which measures the strength of selection against a haplotypic or genotypic class. A selection coefficient of 1 is typical of a fully lethal $(L)$ factor. For overdominance, the fitness 
values for $L L, L l$ and $l l$ are $1-s_{1}, 1$ and $1-s_{2}$, respectively. Similarly, the fitness values for partial dominance for $L L$, $L l$ and $l l$ are $1,1-h$ s and $1-s$.

\section{Rarity of overdominant lethal factors in the embryo lethal system}

Overdominant lethal factors have been rarely detected for the conifer embryo lethal system using dominant marker systems: about one out of 10-20 lethal factors are overdominant (Kuang et al, 1999; Remington and O'Malley, 2000a, b). Overdominant lethal factors may be rarely detected because they are tightly linked to rare marker alleles within coadapted multilocus complexes. This is the case with overdominant lethal factors linked to rare isozyme alleles in marine mollusks (McGoldrick and Hedgecock, 1997) although these findings are controversial (Bierne et al, 1998). Another explanation is that overdominant lethal factors are rarely detected due to transient expression specific to early embryo development (Williams et al, 2001). This is consistent with the concept that overdominant lethal factors persist as a consequence of antagonistic or biphasic selection at different life history stages (eg, Bush and Smouse, 1991; Charlesworth and Charlesworth, 1999).

\section{Overdominant lethal factors cannot account for high genetic loads in pines}

The prevalence of partial dominance in the embryo lethal system is supported by the high genetic loads in conifers (Williams and Savolainen, 1996). Overdominant lethal factors are largely excluded from genetic load estimation, so partially dominant lethal factors must be present if zygotic selection is solely operative. Genetic load is measured in lethal equivalents, 'a group of mutant genes...they would cause on the average one death, eg one lethal mutant, or two mutants each with 50 per cent probability of causing death...' (Morton et al, 1956). In mammals and dioecious plants, lethal equivalent number is estimated from the slope of a log-linear survivorship equation constructed between multiple generations of consanguineous matings. The slope $B$ (or $2 B$ for zygotes) represents the hidden genetic damage that would be expressed fully only in a complete homozygote $(F=1)$. The intercept $A$ estimates the amount of expressed damage in a random mating population.

Dominant loci contribute to the estimate of $B$, but overdominant loci contribute equally to estimates of $A$ and $B$ (Morton et al, 1956). In practice, the value of $A$ also includes environmental contributions to mortality; hence, it does not provide an unbiased estimate of overdominant effects. This holds true for the special case of selfing organisms where the log-linear survivorship equation is estimated within a single generation (Sorensen, 1969; Franklin, 1972). High genetic loads in conifers are attributed to a prevalence of partially dominant effects.

\section{Selection prior to fertilization can bias dominance estimation}

Gametic selection can mimic zygotic selection, biasing estimates of dominance and degree of lethality (Vogl and $X u, 2000)$. Directional gametic selection, meiotic drive and opposing gametic selection all describe cases where an allele is selectively transmitted through one or both gamete types, distorting gametic ratios prior to fertilization. If the allele frequencies are not equal between gamete types at the time of fertilization, the resulting genotypic frequencies will be skewed prior to zygotic selection biasing dominance estimation. Gametic selection must thus be measured separately prior to estimating dominance.

Directional gametic selection mimics apparent partial dominance or underdominance. In this case, selection occurs in the same direction in one or both sexes (Fu and Ritland, 1994b; Fu et al, 1997). Opposing gametic selection mimics overdominance by altering the allelic proportions in opposing directions in each gamete type prior to fertilization (Kidwell et al, 1977). For example, if female gametes have $20 \% A_{1}$ alleles and $80 \% A_{2}$ alleles but male gametes have $80 \% A_{1}$ alleles and $20 \% A_{2}$ alleles, then the probability of same-allele gametes uniting to form a zygote decreases. Opposing gametic selection can be detected at the diploid stage by the presence of unequal marker heterozygote classes $\left(A_{1} A_{2} \neq A_{2} A_{1}\right)$. True overdominance is indicated when the heterozygote classes are equal $\left(A_{1} A_{2}=A_{2} A_{1}\right)$ and genotypic classes adhere to a 1:2:1 ratio before zygotic death to both homozygote classes (Kuang et al, 1999). In conifers, a direct test for opposing gametic selection is straightforward with haploid megagametophytes. The haploid megagametophyte is the functional megaspore for the egg nucleus, so its haplotype is genetically identical to the female gamete's haplotype. If a heterozygous embryo has an $A_{2}$ megagametophyte, then its maternal gamete must also be $A_{2}$. By discerning megagametophytes of heterozygous embryos, the two heterozygote classes can distinguished.

During the lengthy 12-month interval between pollination and fertilization in pines (Gifford and Foster, 1989, pp 432-443), gametic selection seems most likely to occur at two stages: (1) during the 8 weeks after pollination and/or (2) 7-10 days prior to fertilization. The first stage occurs during meiosis in the megaspore mother cell soon after the haploid female gametophyte has started developing. The pollen tube grows toward the megaspore mother cell, exuding secretions which cause cell collapse in maternal sporophytic and gametophytic tissues (Owens and Morris, 1990). This intercellular signaling between pollen and maternal sporophyte is a likely contributor to gametic selection. The second stage occurs 7-10 days before fertilization when ovular secretions also start intercellular signaling, reviving pollen tube development (Takaso and Owens, 1994). Aberrant pollen tube growth and morphology is often observed during this stage, so gametic selection has been hypothesized at this stage as well (Takaso and Owens, 1994).

\section{Polyembryony is not a selective force during pine embryo development}

Polyembryony, or multiple embryos in one ovule, can be discounted as a selective mechanism in pines contrary to brood reduction theory (Haig, 1992). Pinus radiata has on average 2.3 embryos with a range of 1-4 (Lill, 1974); both cleavage and simple polyembryony occur in equal proportions (Burdon and Zabkiewicz, 1973). Cleavage embryos are monozygotic or identical genotypes so type of polyembryony exerts no selection consequences for 
embryo competition. Simple polyembryony refers to embryos that share a common maternal haplotype with different paternal haplotypes due to the fact that each egg cell within the ovule is fertilized by a different pollen grain. There is a high degree of genetic similarity among multiple embryos within an ovule because they share a common haplotype. Likewise, polyembryony does not affect the segregation ratios of embryo lethal factors or molecular markers in the offspring (Hedrick and Muona, 1990), nor can polyembryony be detected experimentally for genetic load estimates. For example, a tree carrying 10 lethals has a $95 \%$ probability of an empty self-fertilized seed for a single fertilized archegonium which is close to 93\% for two fertilized archegonia per ovule (Koski, 1971). Selective consequences of polyembryony are negligible in pines because there are few embryos per ovule, half are derived from cleavage polyembryony and genetic similarity is high for simple polyembryony.

The prevalence of partial dominance does not exclude the presence of overdominance. Zygotic selection in the embryo lethal system may be due to multiple genetic mechanisms, partially dominant and overdominant effects alike. An overdominant genetic mechanism has been hypothesized as one of several means for excluding selfed embryos (Williams et al, 2001). As each zygote develops into a multicellular proembryo, the proembryo's suspensor elongates, pushing the embryo proper into a chalazal direction towards the nutritive tissue of the haploid female megagametophyte (Friedman, 1992). This contact between the embryo proper and its megagametophyte has been hypothesized to signal stage-specific apoptosis, resulting in the death of $95 \%$ of self-pollinated embryos (Koski, 1971).

Molecular marker dissection of the embryo lethal system The rarity of overdominant lethal factors can be tested using genome-wide scans of marker genotypic frequencies. This approach parallels quantitative trait loci (QTL) analysis, except that viability analysis is based on binomial (or multinomial) distributions rather than normally distributed trait means and only surviving embryos are generally assayed. The central assumption to any type of viability analysis is that a marker or marker interval is linked to a single viability locus. A marker allele or haplotype tightly linked to a lethal allele will be absent or under-represented relative to the expected Mendelian ratios of 1:2:1, so that transmission ratio distortion is detected. Distorted genotypic ratios observed in a selfed pedigree provide inference for one or more of the following: degree of dominance, selection coefficients for embryo viability loci and recombination fraction between lethal and marker alleles.

Deducing a genetic model for embryo lethals using genotypic frequencies was first proposed by Sorensen (1969). The advent of molecular markers prompted a maximum likelihood model for embryo viability analysis (Hedrick and Muona, 1990). With this model, the observed frequencies of the three genotypic classes from selfed progeny are used to jointly estimate the selection coefficient and the recombination fraction between the marker and the lethal factor. With only two degrees of freedom, the degree of dominance cannot be estimated; so the simplest case, complete dominance $(h=0)$, is assumed (Hedrick and Muona, 1990). The drawback is that the magnitude of the lethal effects is confounded with recombination fraction estimates between the marker and the lethal factor.

One solution to this drawback is to use the model-free graphical representation method ( $\mathrm{Fu}$ and Ritland, 1994a). This generalized single-marker method infers the degree of dominance by graphically partitioning the selection space among all possible segregation ratios ( $\mathrm{Fu}$ and Ritland, 1994a). The tradeoffs here are that there is overlap in the selection space for different degrees of dominance, and there are no error estimates (Kärkkäinen et al, 1999). With this approach, recombination fractions and selection coefficients between a marker and its putative lethal locus must be assumed (Fu and Ritland, 1994a). Zygotic selection is assumed a priori so bias from gametic selection must be tested separately. Despite these drawbacks, results from graphical representation have been robust when compared to a Bayesian approach (Kärkkäinen et al, 1999).

Mapping lethal factors between flanking markers, or interval mapping, is more powerful than single-marker methods. It provides greater flexibility for a wider range of genetic models as well as superior estimation properties (Fu and Ritland, 1994b; Mitchell-Olds, 1995; Cheng et al, 1996). The degree of dominance, recombination fractions and the selection coefficient(s) can be estimated simultaneously with interval mapping. Maximum likelihood models for inferring zygotic and gametic selection can be compared directly (Cheng et al, 1996). Interval mapping has not been used more widely because there are few saturated linkage maps based on selfed pedigrees. Selfing increases marker homozygosity to the extent that linkage maps based on selfed pedigrees tend to be sparse or of low density (Vogl and Xu, 2000).

This study was based on three cohorts of selfed offspring from a single $P$. taeda parent. Genotypic ratios from codominant microsatellites were used to test four hypotheses about the genetic basis of the embryo lethal system: (1) overdominant lethal factors rarely occur within the embryo lethal system, (2) overdominant lethal factors are rarely detected because they are transient and display stage-specific expression, (3) overdominant lethal factors are rarely detected due to tight linkage with rare marker alleles and (4) dominance estimation is unbiased by gametic selection.

\section{Methods and materials}

\section{Experimental design and DNA sampling}

Pollinations for P. taeda L. parent 7-1037 were conducted using a branch-replicate design (Williams et al, 2001). Self-pollinations were made in each of three branches, each serving as a replicate. On a fourth branch, three outcrossed pollinations were made by bagging strobili and using processed windborne pollen. Insect predation was prevented by caging developing strobili until harvest. This previous study reported that pollination yielded 1805 selfed seeds and 359 outcrossed seeds; the filled seed yield per cone is only $19.8 \%$ per cone for selfing compared to an average of $82.7 \%$ filled seeds per cone for outcrossing (Williams et al, 2001). Based on direct estimates, parent 7-1037 has 7.3 lethals (Williams et al, 2001). One parent of 7-1037 is 7-51 and the other parent is 7-56, unrelated to $7-51$. 
Table 1 Microsatellites used in molecular dissection of a selfed Pinus taeda family (primer sets were previously reported in Auckland et al, 2002)

\begin{tabular}{|c|c|c|c|}
\hline Marker & GenBank accession & Primer & Repeat motif \\
\hline PtTX2031 & AF286613 & $\begin{array}{l}\text { F: 5'-GAAGAAGCTTATGCCTTTAGATGAA-3' } \\
\text { R: 5'-AGCGGGATATTATAGAGTTTGTGTC-3' }\end{array}$ & $(\mathrm{GTGA})_{8} \mathrm{GT}_{14}$ \\
\hline PtTX2034 & AF286614 & $\begin{array}{l}\text { F: } 5^{\prime} \text {-TCTGAGGAGGAACATGTCATTTACT- } 3^{\prime} \\
\text { R: } 5^{\prime} \text {-GCATGTCTGAATTATTGTGTTCTAT- } 3^{\prime}\end{array}$ & $(\mathrm{TTTG})_{9}$ \\
\hline PtTX2080 & AF286600 & $\begin{array}{l}\text { F: 5'-AAAGATGGTCGGTTGTAAAGTT-3' } \\
\text { R: 5'-TTGTCAGGCGGATAAGGTT-3' }\end{array}$ & $(\mathrm{CAT})_{5} \ldots(\mathrm{CGT})_{7} \mathrm{CAT}(\mathrm{CGT})_{2}(\mathrm{CGT})_{7}$ \\
\hline PtTX2085 & AF286601 & $\begin{array}{l}\text { F: 5'-CAAGTCGGATACGCAAACGTT-3' } \\
\text { R: 5'-AGTAGATCCAAGTAAGGGTAAC-3' }\end{array}$ & $(\mathrm{CAA})_{9}$ \\
\hline PtTX2093 & AF286602 & $\begin{array}{l}\text { F: 5'-AATTTGACGGGTTTTAC-3' } \\
\text { R: 5'-GTGGCACATGGATTTCT-3' }\end{array}$ & $(\text { GTTT })_{3}$ GCCTGCTT $(\text { GTTT })_{9} \ldots(\text { GTTT })_{10} \mathrm{~T}_{8}$ \\
\hline PtTX2095 & AF286604 & $\begin{array}{l}\text { R: 5'-TCCGGTATTGTCTCTGTTC-3' } \\
\text { F: 5'-TTCGCAAACGTATCCTAAC-3' }\end{array}$ & $(\text { TTTG })_{15}$ TTTTG $(\text { TTTG })_{3}(\text { TTTG })_{4} \ldots(\mathrm{GT})_{12}$ \\
\hline PtTX3018 & AF286616 & $\begin{array}{l}\text { F: 5'-CCATTTATGAACCAGAGA-3' } \\
\text { R: 5'ATTAAAACCATGAGACCTT-3' }\end{array}$ & $(\mathrm{GAT})_{13}$ \\
\hline PtTX3021 & AF286617 & $\begin{array}{l}\text { F: 5'-TTCATCCTAGCTGCTTGCTTT-3' } \\
\text { R: 5'-CTCAGCGTCTACCCCATCAA-3' }\end{array}$ & $(\mathrm{GTTTTT})_{4} \ldots(\mathrm{GTT})_{5} \ldots(\mathrm{GTT})_{5} \ldots(\mathrm{GTT})_{4}$ \\
\hline PtTX3025 & AF143970 & $\begin{array}{l}\text { F: 5'-CACGCTGTATAATAACAATCTA-3' } \\
\text { R: 5'-TTCTATATTCGCTTTTAGTTTC-3' }\end{array}$ & $(\mathrm{CAA})_{10}$ \\
\hline PtTX3026 & AF143971 & $\begin{array}{l}\text { F: 5'-AATACTTGGGAGGGATAC-3 } \\
\text { R: 5'-AATAGCCAGTTTTGTTTG-3' }\end{array}$ & 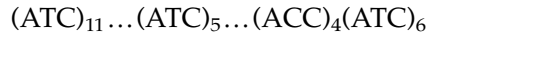 \\
\hline PtTX3034 & AF143974 & $\begin{array}{l}\text { F: 5'-TCAAAATGCAAAAGACG-3' } \\
\text { R: 5'-ATTAGGACTGGGGATGAT-3' }\end{array}$ & $(\mathrm{GT})_{10}(\mathrm{GA})_{13}$ \\
\hline PtTX3044 & AF286605 & $\begin{array}{l}\text { F: 5'-ACCCTTTTGCCCTCACC-3' } \\
\text { R: 5'-TAGCATAATCCACCAGAATAACTC-3' }\end{array}$ & $(\mathrm{ACT})_{42} \mathrm{ATT}(\mathrm{ACT})_{10}$ \\
\hline PtTX3047 & AF286606 & $\begin{array}{l}\text { F: 5'-TTGGAATACTTGCACGATGAC-3' } \\
\text { R: 5'-ATTTAGATAGGAGATGGTTGTTTA-3' }\end{array}$ & $(\mathrm{TACA})_{3}(\mathrm{TA})_{2}(\mathrm{CA})_{20}$ \\
\hline PtTX3049 & AF286607 & $\begin{array}{l}\text { F: 5'-GAAGTGATAATGGCATAGCAAAAT-3' } \\
\text { R: 5'-CAGACCCGTGAAAGTAATAAACAT-3' }\end{array}$ & $(\mathrm{TG})_{16}$ \\
\hline PtTX3063 & AF286610 & $\begin{array}{l}\text { F: 5'-CAATCAGAATCAGCGGCAAACAAA-3' } \\
\text { R: 5'-TTCAACAACATTCATCACACTA-3' }\end{array}$ & $(\mathrm{CAA})_{7}(\mathrm{CAT})_{2}(\mathrm{CAA})_{6} \mathrm{CAG}(\mathrm{CAA})_{15}$ \\
\hline PtTX3068 & AF286611 & $\begin{array}{l}\text { F: 5'-ATAAAATTGAGAAGAAAAAGTT-3' } \\
\text { R: 5'-TTGTTGAATGTAGAATGGTAG-3' }\end{array}$ & $(\mathrm{AAC})_{18}$ \\
\hline PtTX3098 & AF277847 & $\begin{array}{l}\text { F: 5'-TTTGCACTATGGCATAAGTCCT-3' } \\
\text { R: 5'-CCCTGTTTCTACCCTTGATGA-3' }\end{array}$ & $(\mathrm{GTT})_{8}$ \\
\hline
\end{tabular}

Three cohorts of selfed seeds from $P$. taeda parent 7-1037 were separately assayed for 17 microsatellites (Table 1). In the first cohort, 210 mature embryos were excised and destructively sampled for molecular marker dissection. In the second cohort, 173 mature embryos and megagametophytes were destructively sampled to test for gametic selection. Haplotyping megagametophytes from heterozygous embryos provided a direct count for each of the two marker heterozygote classes, defined as $A_{1} A_{2}$ and $A_{2} A_{1}$. In the third cohort, 108 filled seeds were germinated in a Petri dish, and then grown in the greenhouse for testing stage-specific selection (Williams et al, 2001). Seedlings were sampled for DNA after primary needle emergence. Genomic DNA was extracted from needles, embryos and haploid megagametophytes, using a modified Doyle and Doyle (1987) protocol.

\section{Microsatellite data collection, linkage analysis and population survey}

The detailed microsatellite protocols are described in Auckland et al (2002). PCR reactions consisted of $50 \mathrm{ng}$ template DNA, $1 \mathrm{M}$ Tris ( $\mathrm{pH} 9.0), 20 \mathrm{mM}$ ammonium sulfate, $1 \%$ DMSO, $100 \mu \mathrm{g} / \mu \mathrm{l}$ gelatin, $100 \mu \mathrm{M}$ each $\mathrm{dNTP}, 200 \mathrm{nM}$ each forward and reverse primer, $0.625 \mathrm{U}$ Taq polymerase and $2.0 \mu \mathrm{l}$ of $50 \mathrm{mM} \mathrm{MgCl}_{2}$, in a $25 \mu \mathrm{l}$ reaction volume. A Perkin-Elmer 9700 thermal cycler was used for the touchdown PCR protocol. PCR products were assayed with standard PAGE gels or using automated sequencers.

Seven of the 17 microsatellites were assayed using polyacrylamide gels (Williams et al, 2001; Auckland et al, 2002). The remaining 10 microsatellites were assayed using one of two automated methods (Zhou et al, 2002). The first method used the ABI 373 automated sequencer (Applied Biosystems, Foster City, CA, USA) where each primer pair had one of three fluorescent dyes (6-FAM, TET, or HEX) attached to the $5^{\prime}$ end of the forward primers (Applied Biosystems, Foster City, CA, USA). PCR reactions were run separately for each marker, and then $5 \mu \mathrm{l}$ of each PCR product was pooled. The pooled PCR product $(1.5 \mu \mathrm{l})$ was dried and then rehydrated for gel loading with $1.5 \mu \mathrm{l}$ formamide/EDTA loading buffer and $0.5 \mu \mathrm{l}$ GENESCAN 500 TAMRA internal standard. Electrophoresis was performed using 7\% Long Ranger ${ }^{\mathrm{TM}}$ $6 \mathrm{M}$ urea/1 $\times$ TBE gels (FMC BioProducts, Rockland, ME, USA). The detection and sizing of PCR products were performed using GENESCAN 672 (version 1.1). The results from Genotyper (version 1.1.1) (Applied Biosystems) were checked against manual scoring to ensure accuracy. The second method used the LI-COR Model IR 4200 automated DNA sequencer (LI-COR, Inc., Lincoln, NE, USA) with primers directly labeled with fluorescent dyes (Zhou et al, 2002). Gels were $25 \mathrm{~cm}$ in length and $0.25 \mathrm{~cm}$ in thickness, and contained $7 \%$ 
Long-Ranger gel solution (FMC BioProducts, Rockland, ME, USA) and $7 \mathrm{M}$ urea in $1 \times$ TBE buffer. IRD700- and IRD800-labeled sizing standards (50-350 bp) (LI-COR, Lincoln, NE, USA) were used to estimate allele sizes.

Interval mapping hinges on finding linked markers within the selfed pedigree. Linkage analysis was based on the F2 intercross option in MAPMAKER version 3.0 (Lander et al, 1987). For those markers with incomplete phase information, linkage was established using reciprocally coded marker data to represent both repulsion and coupling options. Markers were assigned to linkage groups from a two-point analysis on the basis of LOD $\geqslant 3.0$ and $r<0.4$ as thresholds.

Marker allele frequencies were estimated from a population survey of $130 \mathrm{P}$. taeda samples throughout its natural range (Al-Rababah and Williams, 2002). The four microsatellites used were PtTX3063, PtTX2085, PtTX2093 and PtTX3025. Rare allele frequency was defined as $q<0.05$, and common allele frequency was defined as $q \geqslant 0.05$ (Marshall and Brown, 1975).

\section{Analysis of genotypic frequencies}

Single-marker analysis was conducted as sequential testing: (1) a $\chi^{2}$-test for statistical significance for distorted marker genotypic ratios, (2) estimation of the degree of dominance using the graphical representation approach and (3) a maximum likelihood test for complete dominance. Deviation from the expected 1:2:1 Mendelian ratios were tested with a $\chi^{2}$-test for each microsatellite, using the threshold $\chi^{2}$-value of 10.60. The Bonferroni experiment-wise error rate $\left(\alpha^{\prime}\right)$ was used to adjust the significance threshold upward, so each $\chi^{2}$-test had a significance level of $\alpha=0.005$, but the experimentwise level was $\alpha^{\prime}=0.0817$. A more stringent level of $\alpha^{\prime}=0.0017(\alpha=0.001)$ was also tested.

The degree of dominance for all distorted markers was estimated using the model-free graphical representation method (Fu and Ritland, 1994a). This was followed by a conservative test for complete dominance $(h=0)$ using a maximum-likelihood algorithm for single markers (Hedrick and Muona, 1990) and a LOD threshold of 3.5. If a marker had statistically significant genotypic distortion, showed overdominance for the graphical representation method and had no combination of recombination fraction or selection coefficient which gave a LOD score of 3.5 or higher, then the marker was considered to be linked to an overdominant lethal factor.

For a pair of flanking markers, the expectation/ conditional maximization (ECM) algorithm (Meng and Rubin, 1993) was used to estimate genetic parameters and locus the order of the lethal factor relative to its flanking markers (Cheng et al, 1996; Kuang et al, 1999). The estimated genetic parameters included selection coefficients $\left(s_{1}, s_{2}\right)$ for the lethal factor $(L)$, its degree of dominance $(h)$ and its recombination fractions $\left(r_{1}, r_{2}\right)$ with each flanking marker $(A-L$ or $B-L)$. The relative position of the lethal factor was compared between locus-order models $A-B-L$ vs $A-L-B$.

The ECM algorithm is an extension of the EM algorithm. In the case of overdominance, one cycle of ECM iteration consisted of one E (expectation) step to estimate $a_{i j}$, and two CM (conditional maximization) steps to estimate $r_{1}, r_{2}, s_{1}$ and $s_{2}$ (Meng and Rubin, 1993; Cheng et al, 1996; Kuang et al, 1999). Equations for log- likelihood and maximum likelihood scores have been generalized for the case of codominant markers and overdominant lethal factors in selfed pedigrees (Kuang et al, 1999). When the difference between the value of an estimate in the previous CM-step and the current one was less than 0.001, the ECM iteration was stopped, and the resulting estimates of recombination fractions $\left(r_{1}, r_{2}\right)$ and selection coefficients $\left(s_{1}, s_{2}\right)$ were reported for the overdominant case. The LOD significance threshold of 3.0 was an acceptable threshold for a single pedigree at 10-cM spacing (Lander and Botstein, 1989).

As an example, if two linked markers in coupling $(A B /$ $a b)$ are hypothesized to be linked to a lethal factor $(L)$ in locus order $A-L-B$, then recombination fractions for $A-L$ and $L-B$ can be defined as $r_{1}$ and $r_{2}$. Selection is expressed in terms of relative fitness: in the case of overdominance, relative fitness is 1 for $L l, 1-s_{1}$ for $L L$ and $1-s_{2}$ for $l l$. The nine observed $A B$ marker genotypes for the selfed offspring can be written as the $a_{i}$ matrix where $i=1,2, \ldots, 9$. Note that the $L L, L l$ and $l l$ genotypes can only be inferred via the paired marker loci, so direct counts of the observed nine marker genotype classes $\left(a_{i}\right)$ are used to obtain the expected counts. Assuming that there is no chiasma interference for the coupling-phase segment $A-L-B / a-l-b$, the matrix of expected frequencies for the 27 expected genotypic frequencies is defined as $F_{i j}(i=1, \ldots, 9 ; j=1,2,3)$, and the selection matrix for genotypes $L L, L l$ and $l l$ in the overdominant case is defined as $G_{j}(j=1,2,3)$ for locus order $A-L-B$ (Cheng et al, 1996; Kuang et al, 1999). Using $F_{i j}$ and $G_{i j}$ matrices, the expected counts $\left(a_{i j}\right)$ matrix can be obtained (Equation (1)), where $i=1,2, \ldots, 9$ and $j=1,2,3$ compose all possible $(9 \times 3=27) A-L-B$ genotypes (Cheng et al, 1996; Kuang et al, 1999):

$$
a_{i j}=a_{i} \times \frac{F_{i j} \times G_{j}}{\sum_{k=1}^{3}\left[F_{i k} \times G_{k}\right]}
$$

The locus-order models $A-L-B$ and $A-B-L$ were compared with the observed genotypic frequencies. The model with the smallest $\chi^{2}$-value was accepted as correct. Variances of the ECM estimates were determined by inverting elements of Fisher's information or I matrix (Bailey, 1961; Cheng et al, 1996).

To check for transmission ratio distortion causes other than selfing, a related outbred pedigree with 118 offspring (Zhou et al, 2003) was assayed for those 17 microsatellites which were polymorphic and informative in the outbred pedigree: PtTX2034, PtTX2080, PtTX2093, PtTX3018, PtTX3021, PtTX3026, PtTX3034, PtTX3044, PtTX3049, PtTX3063.

Gametic selection was tested by assaying megagametophytes for the second cohort of 173 selfed offspring. Megagametophytes from the 118 heterozygous embryos were assayed to infer allele frequencies of female gametes, and to infer counts for heterozygote classes $A_{1} A_{2}$ and $A_{2} A_{1}$.

\section{Results}

Overdominant lethal factors were numerous for the $P$. taeda embryo lethal system based on distorted marker genotypic ratios. Seven chromosomal segments with embryo lethal factors were detected using $17 \mathrm{P}$. taeda microsatellites. Each of the seven segments had distorted 
genotypic ratios exceeding a $\chi^{2}$ significance level of 10.60 $(\alpha=0.005)$ (Table 2). Five of these seven were overdominant: PtTX2093, PtTX3025, PtTX3049, PtTX3068 and PtTX2085-3063 (Table 2). Of these, single markers PtTX2093, PtTX3025, PtTX3049 and PtTX3068 were strongly overdominant. The fifth segment was detected with two markers, PtTX2085 and PtTX3063, linked in coupling phase $(r=0.217)$, which allowed the use of the ECM algorithm. Two other chromosomal segments with distorted ratios showed partial dominance (PtTX2095) and underdominance (PtTX3021) (Table 2).

Using the graphical representation method, the same five chromosomal segments were inferred as overdominant (Figure 1; Table 2). The more conservative test, the complete dominance model, could be rejected for four of these five segments (Table 2). The exception was PtTX3025. Although strongly overdominant, a semilethal factor with complete dominance $(s=0.5, h=0)$ appeared to be closely linked to PtTX3025 $(r=0.05)$. This complete dominance model for PtTX3025 was supported by an LOD score of 4.217 (Table 2).

Overdominant lethal factors linked to markers PtTX3049, PtTX3063 and PtTX3068 were consistent for the second cohort of 7-1037 selfed seeds. All the three markers had statistically significant deviations from expected genotypic ratios at $\alpha=0.01$ : PtTX3049 had marker ratios of 28:118:22 with $\chi^{2}=27.95$, PtTX3063 had marker ratios 26:95:52 with $\chi^{2}=9.49$ and PtTX3068 had marker ratios 26:101:36 with $\chi^{2}=10.52$ based on the graphical representation method. The third cohort of selfed seeds also supported overdominance for PtTX3063 although symmetric overdominance was less apparent at this later stage of development $\left(10: 65: 33, \chi^{2}=14.28\right)$

Using the ECM algorithm, symmetric overdominance was detected for PtTX2085-3063 (Table 3). The putative zygotic lethal locus $(L)$ was more closely linked to PtTX3063 $\left(r_{2}=0.0267\right)$ than to PtTX2085. Coding PtTX2085 as locus $A$ and PtTX3063 as locus $B$, locus- order models $A-L-B$ and $A-B-L$ were first estimated using a maximum likelihood model for dominance (Cheng et al, 1996; Kuang et al, 1999). Estimates of $h$ were negative, indicating that overdominance was a better fit than dominance. The $A-L-B$ locus-order model was supported by an LOD value of 3.18 (Table 3 ). The $\chi^{2}$ test for locus order showed that $A-L-B\left(\chi^{2}=6.18\right)$ was a better fit with the observed data than $A-B-L\left(\chi^{2}=7.92\right)$. Selection coefficient estimates were symmetric $\left(s_{1}=0.48\right.$ and $s_{2}=0.44$ ) (Table 3).

Overdominant lethal factors were linked to both common and rare marker alleles. PtTX2085-3063 had alleles that were a mixture of common and rare alleles.

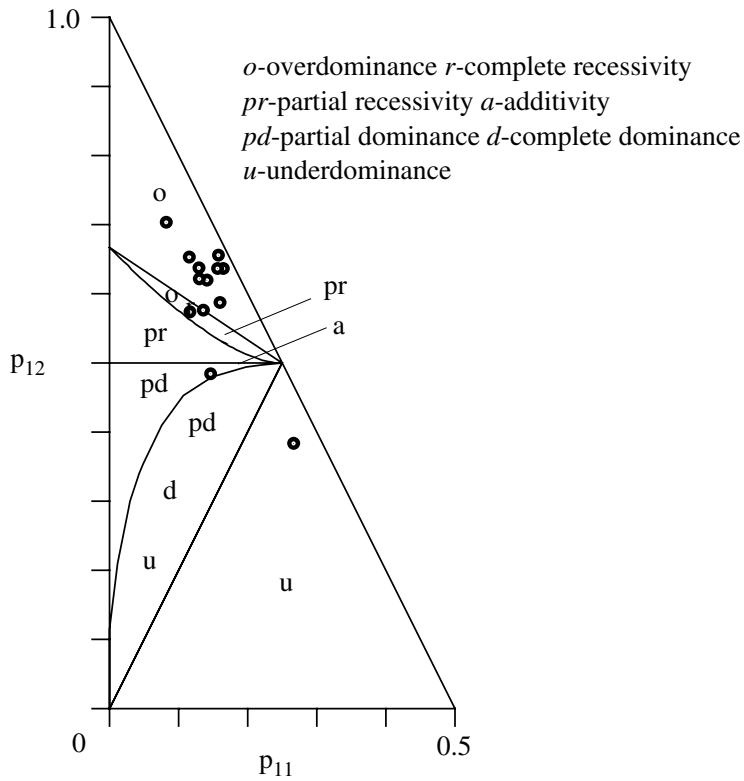

Figure 1 Graphical representation of embryo viability based on a selfed $P$. taeda family and microsatellite markers.

Table 2 Sequential testing for degree of dominance using single markers

\begin{tabular}{|c|c|c|c|c|c|c|c|}
\hline \multirow[t]{2}{*}{ Markers } & \multirow[t]{2}{*}{ GP alleles $(b p)$} & \multirow[t]{2}{*}{ Parent alleles (bp) } & \multicolumn{2}{|c|}{ Embryo maturity } & \multirow[t]{2}{*}{$\chi^{2}$} & \multirow{2}{*}{$\begin{array}{l}\text { Degree of } \\
\text { dominance }\end{array}$} & \multirow{2}{*}{$\begin{array}{c}\text { LOD test } \\
(\mathrm{h}=0)\end{array}$} \\
\hline & & & $\mathrm{N}$ & Segregation & & & \\
\hline PtTX2031 & 190 & 190,163 & 199 & 38:111:50 & 4.11 & - & - \\
\hline PtTX2034 & 170,140 & 166,140 & 199 & 43: 97:59 & 2.67 & - & - \\
\hline PtTX2080 & 144 & 165,144 & 177 & 41:105:31 & 7.28 & - & - \\
\hline PtTX2085 & 206,187 & 194,187 & 176 & $35: 112: 29$ & $13.50^{*}$ & OV & 0.457 \\
\hline PtTX2093 & 337 & 353,337 & 184 & $44: 114: 26$ & $14.04^{* *}$ & OV & 0.004 \\
\hline PtTX2095 & 241,222 & 283,222 & 157 & 58: $76: 23$ & $15.76^{*}$ & PD & -0.338 \\
\hline PtTX3018 & 158,135 & 158,135 & 208 & 51:111:46 & 1.18 & - & - \\
\hline PtTX3021 & 435 & 455,435 & 180 & 48: $69: 63$ & $12.30^{*}$ & UD & -0.080 \\
\hline PtTX3025 & 280,250 & 266,250 & 197 & 23:113:61 & $18.92^{* *}$ & $\mathrm{OV}$ & 4.217 \\
\hline PtTX3026 & 347,335 & 347,335 & 204 & 52:106:46 & 0.67 & - & - \\
\hline PtTX3034 & 208 & 208,203 & 201 & 50:102:49 & 0.06 & - & - \\
\hline PtTX3044 & 390 & 390,284 & 162 & 40: $89: 33$ & 2.18 & - & - \\
\hline PtTX3047 & 361 & 382,361 & 177 & 54: $80: 43$ & 3.00 & - & - \\
\hline PtTX3049 & 322 & 330,322 & 169 & $42: 105: 22$ & $14.68^{* *}$ & $\mathrm{OV}$ & -0.029 \\
\hline PtTX3063 & 315,258 & 315,284 & 182 & $31: 113: 38$ & $11.18^{*}$ & $\mathrm{OV}$ & 1.275 \\
\hline PtTX3068 & 344 & 344,320 & 191 & $26: 110: 55$ & $13.21^{*}$ & OV & 3.122 \\
\hline PtTX3098 & 178 & 189,178 & 163 & 28:96:39 & 6.64 & - & - \\
\hline
\end{tabular}

Markers with distorted segregation ratio are based on the first cohort of selfed Pinus taeda family at the mature embryo stage. Allele sizes in base pairs (bp) are shown for grandparent 7-56 (GP) and parent 7-1037 (P). Homozygote of the larger allele is shown first in genotypic ratios. Statistical significance for $\chi^{2}$ at $\alpha \leqslant 0.005$ is shown in bold as a single asterisk (*) or as a double asterisk (**) for $\chi^{2}$ at $\alpha \leqslant 0.001$. The degree of dominance $(h)$ was determined using the graphical representation method. The conservative LOD test for complete dominance $(h=0)$ was based on maximum likelihood estimation. 
Table 3 Maximum likelihood estimates for $r_{1}, r_{2}, s_{1}$ and $s_{2}$ from interval mapping are shown for the stages of embryo maturity

\begin{tabular}{lllcccrr}
\hline Stage & Best locus order & Model & $\mathrm{r}_{1}( \pm S E)$ & $\mathrm{r}_{2}( \pm S E)$ & $\mathrm{s}_{1}( \pm S E)$ & $\mathrm{s}_{2}( \pm S E)$ & $L O D$ \\
\hline Embryo & $A-L-B$ & OV & $0.1355(0.1081)$ & $0.0267(0.0654)$ & $0.480(0.5973)$ & $0.440(0.6176)$ & 3.18
\end{tabular}

Codes are defined as lethal factor $(L)$, marker PtTX2085 $(A)$, marker PtTX3063 (B) and overdominance $(\mathrm{OV})$.

Table 4 Gametic selection was absent for microsatellites PtTX3049, PtTX3063 and PtTX3068, which were linked to overdominant lethal factors

\begin{tabular}{lcccc}
\hline Marker & $\mathrm{A}_{1} \mathrm{~A}_{2}$ counts & $\mathrm{A}_{2} \mathrm{~A}_{1}$ counts & Allele 1 counts & Allele 2 counts \\
\hline PtTX3049 & 68 & 50 & 96 & 72 \\
PtTX3063 & 47 & 45 & 80 & 82 \\
PtTX3068 & 54 & 46 & 73 & 97
\end{tabular}

Counts for heterozygote classes $\left(A_{1} A_{2}, A_{2} A_{1}\right)$ and allele frequencies were similar; differences were not statistically significant at the $5 \%$ level. The first heterozygote class $A_{1} A_{2}$ refers to a heterozygote where the maternal parent contributed Allele 1 and the paternal parent contributed Allele 2.

The frequencies for PtTX2085 alleles 194 and $187 \mathrm{bp}$ were 0.143 and 0.259 , respectively. Allele $284 \mathrm{bp}$ for PtTX3063 was also common $(q=0.114)$. Similar results were found for two other markers linked to overdominant lethal factors: PtTX2093 had two common alleles ( $q=0.157$ for both alleles) and PtTX3063 had one common allele $(266 \mathrm{bp}, q=0.159)$ and one rare allele (250 bp, $q=0.009)$.

Distorted ratios observed in the selfed offspring were not distorted in the related outbred pedigree. Of the 17 microsatellites, 10 could be checked for transmission ratio distortion in the outbred pedigree: PtTX2034, PtTX2080, PtTX2093, PtTX3018, PtTX3021, PtTX3026, PtTX3034, PtTX3044, PtTX3049 and PtTX3063. None of the markers linked to lethal factors had distorted ratios in the outbred pedigree. The two markers with distorted ratios in the outbred pedigree, PtTX3026 $\left(\chi^{2}=35\right)$ and PtTX3044 $\left(\chi^{2}=9.4\right)$, adhered to expected Mendelian ratios in the selfed pedigree.

Gametic selection was not detected in this study and thus did not bias the estimates of overdominant zygotic selection. Gametic selection was tested for three markers linked to overdominant lethal factors: PtTX3049, PtTX3063 and PtTX3068. In each case, the two heterozygote classes had similar counts, so there was no support for opposing gametic selection as a source of bias (Table 4). Similarly, allele frequencies among gametes did not differ at the 5\% level; hence, meiotic drive was not observed for female gametes either (Table 4).

The overdominant factor linked to PtTX3063 continued to exert an effect throughout early development, persisting to early seedling development. Genotypic ratios for greenhouse seedlings were 10:65:33, with a $\chi^{2}$ value of 14.28. Transient expression of an overdominant lethal factor did not explain the large number of apparent overdominant factors.

\section{Discussion}

Overdominant lethal factors were commonly detected in this study, contrary to previous reports. Methods of viability analysis produced similar results; overdominant factors were detected with both single-marker methods and interval mapping. Overdominant lethal factors could not be explained by stage-specific expression or biphasic models where one of two opposing selective forces dissipate early, leaving unidirectional selection at later stages (Charlesworth and Charlesworth, 1999). This contrasts with previous findings where an overdominant lethal factor did display stage-specific overdominance (Williams et al, 2001). Rarity of overdominant lethal factors did not occur as a result of cosegregation with rare marker alleles. Similarly, the detection of overdominant lethal factors could not be explained by a bias from gametic selection. Other causes of transmission ratio distortion expressed in a related outbred pedigree had no bearing on the patterns of distorted genotypic ratios observed in the selfed pedigree.

The number of overdominant lethal factors in this study is incongruent with results from two previous studies on North American hard pine species (Kuang et al, 1999; Remington and O'Malley, 2000a). These genome-wide scans were based on dominant RAPD or AFLP markers (Kuang et al, 1999; Remington and O'Malley, 2000a). In the first study, only one of nine lethal factors for $P$. radiata has symmetric overdominance, but the degree of dominance is uncertain for other lethal factors with low negative $h$ values (Kuang et al, 1999). In the second study, only two of 20 lethal factors showed overdominance in the $P$. taeda study (Remington and O'Malley, 2000a). By contrast, this study had detected four out of seven lethal factors as overdominant.

These incongruent results have at least three explanations: (1) overdominant lethal factors are the result of multiple lethal factors linked to one marker or marker interval, (2) there is inherent bias in the genetic models used with dominant marker systems for inferring the degree of dominance and (3) microsatellites and dominant marker systems sample lethal factors in different parts of the leviathan pine genome. All the three explanations require revised genetic models for the embryo lethal system, and all point to the strong possibility of pseudo-dominance and the need for better fine-mapping tools for viability analysis. The high numbers of overdominant factors in this study are best explained by model constraints inherent to molecular marker dissection methods.

The first explanation is that use of low-density mapping in selfed pedigrees violates the assumption of one lethal factor per marker, biasing estimates of dominance ( $\mathrm{Fu}$ et al, 1997). The presence of the underdominant lethal factor linked to marker PtTX3021 supports this explanation because inbreeding depression cannot be caused by underdominance, suggesting an incorrect genetic model (Fu et al, 1997). This would occur if multiple lethal factors, linked in repulsion phase, cluster in certain chromosomal regions. If so, the embryo lethal system may be composed of balanced lethal 
complexes dispersed across the genome, an idea that has some experimental support (Remington and O'Malley, 2000a; Williams et al, 2001). For example, the true locus-order model might be $A-L_{1}-B-L_{2}-C$, where two lethal factors $L_{1}$ and $L_{2}$ are linked in repulsion as part of a balanced lethal complex, that is, pseudo-overdominance. Each lethal factor is dominant, not overdominant, if analyzed separately. In the absence of polymorphic markers $B$ and $C$, single-marker analysis proceeds for the sparse-marker model $A-L_{1}-L_{2}$, detecting the apparent overdominance caused by pseudooverdominance. Fine-scale mapping of genotypic frequencies for codominant markers $A, B$ and $C$ should detect partially dominant rather than overdominant lethal factors.

Multiple balanced lethal complexes also explain why high-density mapping with AFLP and RAPD markers detected a similar number of lethal factors as this study. In this study, nearly one lethal factor was detected for every two microsatellites, an order of magnitude higher than the two high-density mapping studies. Nine lethal factors were detected with 150 RAPD markers for $P$. radiata (Kuang et al, 1999) and 20 lethal factors were detected with 226 AFLP markers in P. taeda (Remington and O'Malley, 2000a). Perhaps detection of lethal factors appears to be curvilinear to marker number because lethal factors are clustered into balanced lethal complexes along certain chromosomal segments rather than randomly distributed throughout the genome. Additional markers simply increase the mapping resolution around the balanced lethal complexes, shifting the detection from overdominance to partial dominance, an effect akin to fine-scale mapping.

The second explanation is that detection of true overdominant lethal factors in genome-wide scans was omitted in previous studies due to genetic model constraints inherent to dominant marker systems. Dominant AFLP or RAPD markers are scored as band-present $(A, B, C)$ or band-absent $(a, b, c)$. Linkage phase is determined for each pair of loci, unlike pedigrees based on inbred lines. In this approach, only markers linked in coupling which show an excess of band-present individuals are defined as cosegregating with lethal factors. This constraint is due to a limited power for detecting lethal factors in repulsion phase using dominant marker analysis (Kuang et al, 1999; Remington and O'Malley, 2000a). Computer simulation is needed to determine the extent of this linkage-phase bias given a preponderance of balanced lethal complexes.

The third explanation seems to be the least likely: the higher number of lethal factors per microsatellite is due to nonrandom genomic sampling by one or both marker systems. Triplet-repeat microsatellites (Table 1), particularly those from conserved portions of the genome, are often proximal to genomic regions rich in protein-coding genes or regulatory elements (Gerber et al, 1994). The probability of detecting a lethal factor with a single marker will be higher if the marker is closely linked to a defective protein-coding region or a cis-binding regulatory element. Conversely, RAPDs and AFLP markers may selectively sample heterochromatic regions which are often depauperate for genic regions in large plant genomes (McCombie et al, 2000). Dominant marker systems may simply sample the entire genome more randomly than microsatellites.
Our conjecture is that the embryo lethal system is composed of balanced semilethal complexes or an admixture of genetic mechanisms, some of which have an overdominant mode of gene action. In either case, purging will not reduce genetic load due to clustered lethals bound by tight linkage or persistent polymorphism caused by a few loci with symmetric overdominance. Determining the genetic basis for the conifer embryo lethal system requires enhanced statistical and molecular resolution, a serious challenge given the size of this plant genome and the paucity of its genomic resources.

\section{Acknowledgements}

This work is dedicated to the fine scholarship of Dr David L Bramlett. The sponsors were the Duke University's Center for Global Change, Plum Creek Timber Company and Texas Higher Education Coordinating Board ATP Grant \# 999902-090 (to CGW). Special thanks are due to Dr Zhou Yi for automated microsatellite data collection, Dr Yong-Bi Fu for the graphical representation files, Dr David Remington for constructive review and Dr Hanhui Kuang for assistance with the ECM algorithm. The instrumentation for automated marker genotyping was provided by NSF DBI 9871390 to the Institute of Plant Genomics, Texas A\&M University.

\section{References}

Al-Rababah M, Williams CG (2002). Population dynamics of Pinus taeda L. based on nuclear microsatellites. Forest Ecol Manage 163: 263-271.

Auckland LD, Bui T, Zhou Y, Shepherd M, Williams CG (2002). Conifer Microsatellite Handbook. Corporate Press: Raleigh, NC. p 57.

Bailey NTJ (1961). Mathematical Theory of Genetic Linkage. Oxford University Press: Amen House, London.

Bierne N, Launey S, Nacriri-Graven Y, Bonhomme F (1998). Early effect of inbreeding as revealed by microsatellite analyses on Ostrea edulis larvae. Genetics 149: 1893-1906.

Bramlett DL, Popham TW (1971). Model relating unsound seed, embryonic lethals in self-pollinated pines. Silvae Genet 20 192-193.

Burdon RD, Zabkiewicz JA (1973). Identical, non-identical seedling twins in $P$. radiata. Canad J Bot 51: 2001-2004.

Bush RM, Smouse PE (1991). The impact of electrophoretic genotype on life history traits in Pinus taeda. Evolution 45: 481-498.

Charlesworth B, Charlesworth D (1999). The genetic basis of inbreeding depression. Genet Res 74: 329-340.

Cheng R, Saito A, Takano Y, Ukai Y (1996). Estimation of the position, effect of a lethal factor locus on a molecular marker linkage map. Theor Appl Genet 93: 494-502.

Doyle JJ, Doyle JL (1987). A rapid DNA isolation procedure for small quantities of fresh leaf tissue. Phytochem Bull 19: 11-15.

Franklin EC (1969). Inbreeding depression in metrical traits of loblolly pine (Pinus taeda L.) as a result of self-pollination. Technical Report No. 40, School of Forest Resources, North Carolina State University, Raleigh, NC.

Franklin EC (1972). Genetic load in loblolly pine. Am Nat 106 262-265.

Friedman WE (1992). Double fertilization in nonflowering plants and its relevance to the origin of flowering plants. Int Rev Cytol 140: 319-355.

Fu YB, Charlesworth D, Namkoong G (1997). Point estimation and graphical inference of marginal dominance for two viability loci controlling inbreeding depression. Genet Res 70: 143-153. 
Fu YB, Ritland K (1994a). Evidence for the partial dominance of viability of viability genes contributing to inbreeding depression in Mimulus guttatus. Genetics 136: 323-331.

Fu YB, Ritland K (1994b). On estimating the linkage of marker genes to viability genes controlling inbreeding depression. Theor Appl Genet 88: 925-932.

Gerber HP, Seipel K, Georgiev O, Hofferer M, Hug M, Rusconi S et al (1994). Transcriptional activation modulated by homopolymeric glutamine and proline stretches. Science 263: 808-811.

Gifford EM, Foster AS (1989). Morphology and Evolution of Vascular Plants. W.H. Freeman Company: New York.

Griffin RA, Lindgren D (1985). Effect of inbreeding on production of filled seed in Pinus radiata - experimental results and a model of gene action. Theor Appl Genet 71: 334-343.

Haig D (1992). Brood reduction in gymnosperms. In: Elgar MA, Crespi BJ (eds) Cannabalism, Ecology and Evolution. Oxford University Press: London. pp 62-84.

Hedrick PW, Muona O (1990). Linkage of viability genes to marker loci in selfing organisms. Heredity 64: 67-72.

Kärkkäinen K, Kuittinen H, van Treuren R, Vogl C, Oikarinen S, Savolainen O (1999). Genetic basis of inbreeding depression in Arabis petraea. Evolution 53: 1354-1365.

Kidwell JF, Clegg MT, Prout T (1977). Regions of stable equilibria for models of differential selection in the two sexes under random mating. Genetics 85: 171-183.

Koski V (1971). Embryonic lethals of Picea abies and Pinus sylvestris. Commun Inst Forest Fennica 75: 1-30.

Kuang H, Richardson TE, Carson SD, Bongarten BC (1999). Genetic analysis of inbreeding depression in plus tree 850.55 of Pinus radiata D. Don. II. Genetics of viability genes. Theor Appl Genet 99: 140-146.

Lander ES, Botstein D (1989). Mapping Mendelian factors underlying quantitative traits using RFLP linkage maps. Genetics 121: 185-199.

Lander ES, Green P, Abrahamson J, Barlow A, Daly M, Lincoln S, Newburg L (1987). MAPMAKER: an interactive computer package for constructing primary genetic linkage maps of experimental and natural populations. Genomics 1: 174-181.

Lill BS (1974). Development of the female cone of Pinus radiata D. Don. Ph.D. dissertation. University of Canterbury, New Zealand.

Mangelsdorf AC (1952). Gene interaction in heterosis. Heterosis. Iowa State College Press: Ames, Iowa. pp 320-329, 552pp.

Marshall DR, Brown AHD (1975). Optimum sampling strategies in genetic conservation. In: Frankel OH, Hawkes JG (eds) Crop Genetic Resources for Today and Tomorrow. Cambridge University Press: Cambridge. pp 53-80.
McCombie WR, de la Bastide M, Habermann K, Parnell L, Dedhia N, Gnoj L et al (2000). The complete sequence of a heterochromatic island from a higher eukaryote. Cell 100: 377-386.

McGoldrick DJ, Hedgecock D (1997). Fixation, segregation and linkage of allozyme loci in inbred families of the Pacific oyster Crassostrea gigas (Thunberg): implications for the causes of inbreeding depression. Genetics 146: 321-334.

Meng X-L, Rubin DB (1993). Maximum likelihood estimation via the ECM algorithm: a general framework. Biometrika 80: 267-278.

Mitchell-Olds T (1995). Interval mapping of viability loci causing heterosis in Arabidopsis. Genetics 140: 1105-1109.

Morton NE, Crow JF, Muller HJ (1956). An estimate of the mutational damage in man from data on consanguineous marriages. Proc Natl Acad Sci USA 42: 855-863.

Namkoong G, Bishir J (1987). Frequency of lethal alleles in forest tree populations. Evolution 41: 1123-1127.

Owens JN, Morris SJ (1990). Cytological basis for cytoplasmic inheritance in Pseudotsuga menziesii. I. Pollen tube and archegonial development. Am J Bot 77: 433-445.

Remington D, O'Malley DM (2000a). Whole-genome characterization of embryonic stage inbreeding depression in a selfed loblolly pine family. Genetics 155: 337-348.

Remington D, O'Malley DM (2000b). Evaluation of major genetic loci contributing to inbreeding depression for survival and early growth in a selfed family of Pinus taeda. Evolution 54: 1580-1589.

SorensEn FC (1969). Embryonic genetic load in coastal Douglas fir, Pseudotsuga menziesii var. Menziesii. Am Nat 103: 389-398.

Stuber CW, Lincoln SE, Wolff DW, Helentjaris T, Lander ES (1992). Identification of genetic factors contributing to heterosis in a hybrid from two elite maize inbred lines using molecular markers. Genetics 132: 823-839.

Takaso T, Owens JN (1994). Effects of ovular secretions on pollen in Pseudotsuga menziesii (Pinaceae). Am J Bot 81: 504-513.

Vogl C, Xu S (2000). Multiple point mapping of viability and segregation distorting loci using molecular markers. Genetics 155: 1439-1447.

Williams CG, Savolainen O (1996). Inbreeding depression in conifers: implications for breeding strategy. Forest Sci 42: 102-117.

Williams CG, Zhou Y, Hall SE (2001). A chromosomal region promoting outcrossing in a conifer. Genetics 159: 1283-1289.

Zhou Y, Bui T, Auckland LD, Williams CG (2002). Direct fluorescent primers are superior to M13-tailed primers for automated genotyping of Pinus taeda microsatellites. Biotechniques 32: 46-52.

Zhou Y, Gwaze DP, Bui T, Reyes-Valdés MH, Williams CG (2003). No clustering for linkage map based on low-copy and undermethylated microsatellites. Genome 46: 1-8. 